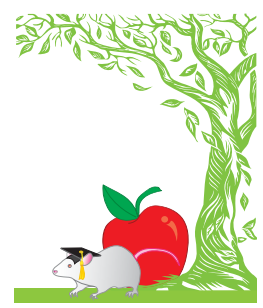

\title{
Engaging students to participate
}

As trainers, we want our sessions to be lively and interactive. We want our students to respond and contribute. We want to be sure that the material we present is processed and applied. Why would we train if the instruction didn't result in subsequent improvements in laboratory animal research tasks? That would be a waste of resources.

But in reality, we sometimes look around our classrooms and see tired faces. We see heads bowed, attention directed at mobile phones instead of PowerPoint slides. No matter how recently updated or relevant it is, the presentation on the screen is not attracting the attention that was intended. How can we capture and keep students' interest?

We must recall that adults learn differently from children. Principles of adult learning, or andragogy, as proposed by Professor Malcolm Knowles, tell us that motivation in training situations should be oriented toward the learner rather than toward the content. Adult students need to appreciate why they are in the classroom and to find reasons for participating in a training session. Simple statements of learning objectives are generally not sufficient to engage adult learners, but interaction between trainers and students is more likely to achieve the intended results. Interactions such as asking questions of the group, conducting exercises in break-out sessions and demonstrating tasks can encourage students to engage with the material. Long ago, Confucius said, "I hear and I forget. I see and I remember. I do and I understand." These observations hold true today, even in a world with many distractions.

So I wanted to share my experience with a tool that trainers can use to turn distractions-specifically mobile phones and other wireless devices-into opportunities for interaction. It's a product called Poll Everywhere (http://www.polleverywhere. com) that energizes the classroom by enabling students to participate in surveys and tracking their responses in real time.

To use Poll Everywhere, a trainer begins by constructing a survey question, testing it out and then incorporating it into a PowerPoint slide. During the classroom presentation, the slide will appear, displaying the question along with instructions for responding, and the trainer can direct students to use their mobile phones or wireless devices to respond to the question. As responses are received, results are presented in a chart on the slide and updated in real time, almost immediately. I've included two examples: one from my lab animal class (course number AHS 369) and another from the American Association for Laboratory
Animal Science's Institute for Laboratory Animal Management program.

When using traditional methods of surveying students, such as asking for a show of hands, responses may be guarded as students look around at their peers to see if their answers match. With Poll Everywhere, trainers can choose to make all responses anonymous and use a counter to track the number of responses. As the number climbs, almost everyone engages and watches as the results are displayed. Students seem less inhibited to participate.

Trainers can ask all kinds of questions, which can be multiple choice or allow short, free-form text responses: "How familiar are you with covered species?" or "Have you experienced a challenge when conducting a euthanasia task?" or "How many digits are on a mouse's forepaw?" Students can share their answers and learn from the responses of their classmates, while trainers can evaluate the group's overall knowledge and pinpoint topics that might need to be revisited or clarified.

The Poll Everywhere site includes tutorials and instructions for using the product. Polls can be saved and reused. Usage is free of charge for fewer than 40 respondents.

By encouraging student participation and engagement, Poll Everywhere can help turn mobile phones from distractions into effective training tools.
I'm taking 369 because

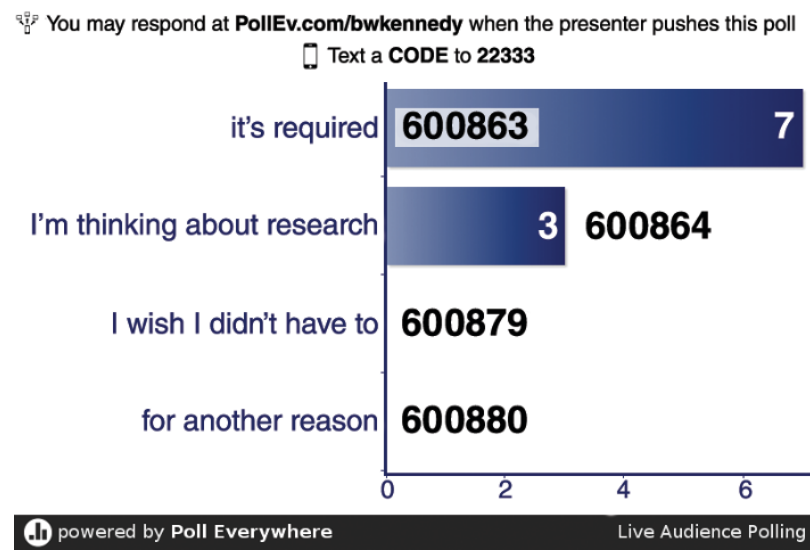

Every manager is a trainer

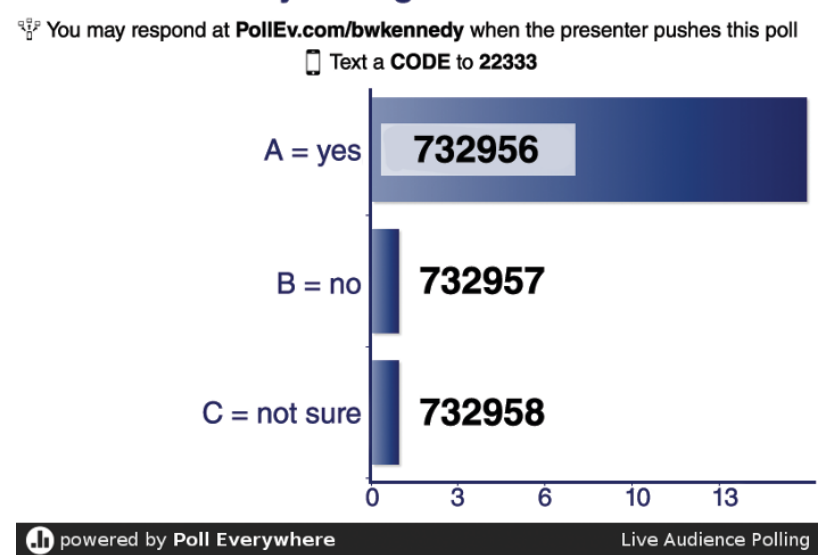

